\title{
Blind Image Deblurring using Bayesian Approach on Parallel Architecture
}

\author{
Naazish Rahim \\ Computer Science \& \\ Engineering \\ Government Engineering \\ College \\ Ajmer(Rajasthan),India
}

\author{
Rakesh Rathi \\ Computer Science \& \\ Engineering \\ Government Engineering \\ College \\ Ajmer(Rajasthan),India
}

\author{
Sudhir Kumar Meesala \\ Computer Science \& \\ Engineering \\ Chouksey Engineering College \\ Bilaspur \\ Bilaspur(C.G), India
}

\begin{abstract}
The objective of image restoration is to reconstruct the primitive scene from a degraded contemplation. This retrieval process is sequential and pivotal to numerous image processing applications. Although classical image restoration has been thoroughly studied $[1,2,3]$ but no one conceived it from parallel computing procedure. Blind image revival is deliverance of estimating the primitive image from the degraded image using partial information about the imaging system. In classical linear image restoration, the blurring function is given, and the degradation course is overturned using one of the many known restoration algorithms. Regrettably, in many pragmatic circumstances, the blur is often unspecified, and minor information is accessible about the primitive image. Therefore, the primitive image $\boldsymbol{F}(\boldsymbol{x}, \boldsymbol{y})$ must be identified directly by using partial or no information about the blurring process and the true image. We pose a novel algorithm for blind image deblurring from a single image using Bayesian and parallel computation. The blur point spread function (PSF) is assumed uniform. We divide the image and exert the algorithm on each part parallelly.
\end{abstract}

\section{Keywords}

Point Spread Function, blurred image, degradation.

\section{INTRODUCTION}

There exist many mundane state of affairs such as astronomy, remote sensing, medical imaging, military detection, public security, and video technology, images are the principal informant. Due to some exposition, ascertained images are debauched. The degradations are primerly incited by blur and noise. The intent of image restoration is to acquire restored image which should approximate nigh to the primitive image. Many works on image

restoration have been reported [4] [5]. The quintessential theory of restoration of a unique image from linear blur and additive disturbance has drawn a lot of research attention [6]. In the single image restoration theory, three notable and distinct procedures are extensively used in order to get practical restoration algorithms: 1) maximum likelihood (ML) estimator 2) maximum a posteriori (MAP) probability estimator and 3) projection onto convex sets (POCS) approach. The persistent development of computer architecture in contemporary years has led to a proliferation interest in image restoration theory. The main courses are unconventional treatments to the classic predicament and looking at novel restoration problems, allowing for more convoluted and computationally intensive algorithms [7, 8]. The restraints of the cited two concepts of algorithms are highly ordered computation and deplete local information for image restoration. Pertinence of such restoration mode arise in the following areas [1].

1) Remote sensing: where several images of the same area are given, and an improved resolution image is sought.

2) Frame freeze in video: where typical individual frame in video signal is generally of abysmal quality and is not desirable for hard-copy printout. Amelioration of a freeze image can be done by using numerous successive images merged together by a superresolution algorithm.

3) Medical imaging (CT, MRI, ultrasound, etc.): these equip the acquisition of several images, yet are curbed in resolution quality. Analytically designed dictionaries, such as DCT, wavelet, curvelet, and contourlets, share the advantages of fast implementation; however, they lack the adaptivity to image local structures [10].

\section{BLIND IMAGE RESTORATION 2.1 Review Stage}

Sub Blind image restoration bargains with estimation of primitive image from observed blurred, degraded image using partial information about the imaging system. Blind deconvolution is a well-known ill-posed impasse, and deblurring course within this formulation aims at reacquiring images with missing crucial frequency details and the uniqueness, stability of solution is not solitary. To address this problem, some methods first estimate the kernel $\mathrm{K}$ and then employ non-blind deconvolution algorithm to fetch the latent image, while others estimate kernel $\mathrm{K}$ and latent image I simultaneously [4]. With known or well estimated kernel, there exist numerous algorithms that can recover the blurred image well . If the kernel can be estimated, we can employ these non-blind deconvolution methods to obtain high-quality latent image. Prior gradient distribution of nature images and sophisticated machine learning algorithm are utilized for kernel estimation in [7]. However, rich and specific information contained in a blurred image is not exploited for estimating deblur kernel. In [9] a method that utilizes additional information from a pair of blurred and noisy images is proposed to estimate kernel. An algorithm that models the deblurred blur kernel and image with wavelets and sparsity constraints has demonstrated promising results [4]. Nevertheless, this procedure does not exploit rich information 
contained in the blurred image as the generic bases are used. In [9], the motion blur point spread function (PSF) is represented in terms of the camera's angular velocity, which is in turn approximated by a linear function of time. Edge spread functions are estimated from isolated edges within the image, and the information along different directions is combined in an optimization procedure to find the best matching PSF.

\subsection{Related work:}

There have been a number of methods proposed in the literature for blind image deconvolution . In [11], Ramya et al concede the problem with inference of the known parameters PSF, Blur length, Blur angle and type of noise, needed for blurring an image. First they apply their degradation model on the input image and use canny filter to detect the edges from blurred image. In [12] Jiange and Wange clutch the problem from two nonnegative distributions, the discrepancy measure consistent with Csiszar's axioms or generalized Kullback distance. In [13] ,Li et al , proposed statistical method is proposed for deblurring two-tone images, i.e., images with two unknown grey levels, that are blurred by an unknown linear filter. The key idea of the proposed method is to adjust a deblurring filter until its output becomes two tone. Two optimization criteria are proposed for the adjustment of the deblurring filter. A three-step iterative algorithm (TSIA) is also proposed to minimize the criteria however none of the algorithm is suitable for parallel architecture.

\subsection{The Proposed Work}

If the convolution operator is non-local, the blurred image counts on the scenery outside the field of panorama. Ignoring this dependency leads to image distortion known as boundary effect. In this algorithm, we rekon two approaches to treat the non-locality. One is to estimate the image extended outside the field of panorama and the other is to treat the influence of the out of view scenery as boundary clutter to deblur image .First approach is considered from the Bayesian point of view and other from singular value decomposition. In the Bayesian statistics, all variables are modeled as random variables with corresponding probability distributions.

We assume that these distributions are absolutely continuous with respect to the Lebesgue measure, thus being characterized by their probability densities functions (PDF). Consider an inverse problem recast in the form of statistical inference: given the observed value of a random variable $b$ that is related through a mathematical model to another random variable $\mathrm{x}$, what can be said about the distribution of the values of $\mathrm{x}$ based on the observation. The key is the formula for the joint probability distribution

$$
\pi\left(x_{x} b\right)=\pi_{p r}(x) \pi(b \mid x)
$$

where $\pi_{p r}(x)$ is the prior density, expressing our knowledge or belief of how the values of $\mathrm{x}$ are distributed prior to the measurement of $b$, while $\pi(b \mid x)$ is the likelihood density, expressing the distribution of the values of $b$ assuming that $\mathrm{x}$ is known.

1. Image Model for kernel assessment assuming uniform blur distribution avoiding non linearity.

$$
\text { a. } G(i, j)=K\left(i_{i} j\right) * F\left(i_{j} j\right)+N\left(i_{j} j\right)
$$

b. Discrete Fourier transform can be used to yield frequency domain model

$$
G(m, n)=K(m, n) F(m, n)+N(m, n) \text { where } \mathrm{m} \text { and } \mathrm{n}
$$
discrete horizontal and vertical frequency variable. c. Inverse procedure will compute
$G(m, n) K-1(m, n)=F(m, n)+N(m, n) k-1(m, n)$

2. Input an blur image.

3. Kernel estimation assuming uniform blur using Bayesian approach.

a. The image is partitioned into smaller frames each of which is large enough to contain information for kernel estimation. Since our blur is uniform so we can process each part individually and estimate the $\mathrm{N}$ and $\mathrm{K}$. The whole image will contain same information so this part will make algorithm computationally efficient and suitable for parallel computation. The following model, after partition holds approximately:

$$
G_{i}\left(x_{x} y\right)=K\left(x_{s} y\right) F_{i}\left(x_{v} y\right)+N_{i}\left(x_{v} y\right)_{s} i=1,2,3 \ldots \times n_{p}
$$

where ${ }^{n_{p}}$ is the total number of partitioned subsections.

b. The blurred image is treated as Gauss-Markov Random Field with the values at any site/pixel $s=\left(i_{s} j\right)$ dependent on its neighbors. Thus, the prior distribution for the image is $P(G)$ proportional to

$\exp \left\{\frac{-1}{2 * \sigma^{2}} \sum_{\text {sites } s \mathrm{t}}(G(t)-G(s))^{2}\right\}$

c. The likelihood probability of the data GM (GaussMarkov) given the image $G_{x} P(G M \mid G)$ proportional to

$$
\exp \left\{\frac{-1}{2 * \sigma_{c}^{2}} \sum_{\text {sites } s}\left[(G(s)-k G(\text { neighbours of } s)]^{2}\right\}\right.
$$

where ${ }^{\sigma}, \sigma_{c}^{2}$ and $\hbar$ are control of smoothness, degree of sharpness and statistical constant.

d. The likelihood probability $P(G M \mid F)$ is calculated in second step so is calculated by Posterior $\propto$ Prior $\times$ Likelihood probability

$$
\begin{gathered}
\operatorname{logp}\left(F^{s} \mid G M\right)=\text { Const }^{s}-\left\{\frac{-1}{2 * \sigma^{2}} \sum_{\text {sites gst }}[G(t)-G(s)]^{2}\right\} \\
-\left\{\frac{-1}{2 * \sigma_{e}^{2}} \sum_{\text {sitess }}\left[(G(s)-k G(\text { neighbours of } s)]^{2}\right\}\right.
\end{gathered}
$$

e. In order to draw information from, we use following algorithm:

1. Draw a new candidate fnew for using random sampling.

2. Compare the posteriors probability postprob = $p(G \mid G M)$ with the old [choose pixel] and the new [neighbors pixel] ${ }^{G}$.

3. If the new value has higher posterior, means it contains more information then noise and accept it. If lower, then accept it with probability $=$ postprob_new / postprob_old

f. The computed $\mathrm{F}^{\prime}$ can be again feeded back to input as a blur image. This process can be repeated many times until we get $F^{\prime}$ close enough to $F$.

4. Regularization, ill condition problem is converted into well conditioned problem with the imposition of constraint to 
reduce ring. The image restoration problem is in general ill conditioned; a small perturbation of the given data produces large deviations in the resulting solution. Since the process of deconvolution attempts to restore the image by some method of inversion, the problem is often ill-conditioned owing to the existence of the additive noise. The direct inverse of the blur transfer function usually has a large magnitude at high frequencies, so excessive amplification of the noise at these frequencies results.

$$
\text { a. Instead of minimizing } \min _{f}\|K f-g\| \text {, we try }
$$
to minimize

$$
\min _{f}\|K f-g\|^{2}+\alpha\|f\|^{2}
$$

where $f$ and $g$ are converted column vector of $F$ and $G$ and some statistical constant $\alpha$. This process eliminates higher frequencies from Noise.

b. Compute the singular value decomposition (SVD) of K:

$$
K=U D V
$$

where $\mathrm{U}, \mathrm{V}$ are orthogonal matrices, and $D=\operatorname{diag}\{d 1, d 2 \ldots \ldots \ldots d N\}$ consists of singular values of $\mathrm{K}$.

c. Compute F using FFT multiplication

$$
\begin{aligned}
& K^{-1}=W^{*} D W \\
& F=W^{*} D W G
\end{aligned}
$$

where $\mathrm{W}^{*}$ is conjugate of $\mathrm{W}$

5. The 3rd and 4th part of this algorithm can repeated iteratively on their respective processor until we get close approximation of image F.

\section{Combine all the images}

\section{Result}

\section{IMPLEMENTATION AND RESULT}

Comparision of the average consumed time is shown as the table 1 .

\begin{tabular}{|l|l|l|}
\hline Deblurring mode & Thread Number & Consumed Time \\
\hline Sequential & One & $5.78 \mathrm{~s}$ \\
\hline Parallel & Four & $1.445 \mathrm{~s}$ \\
\hline
\end{tabular}

From the above experimental results we can know that this method can effectively improve the quality of deblurred image.

In order to achieve the parallelization, we divided the image and call the deblurring function separately from each other however to achieve the parallelization, we can use Haskell matlab package.

The below images represent the result of degradation model, First image represented the original image in Figure 1.

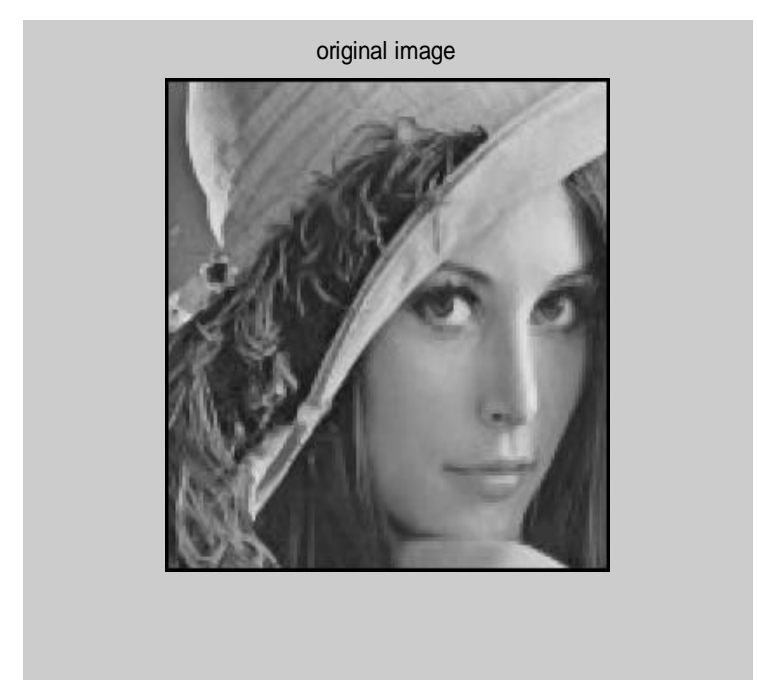

Figure 1 Original Image

We break the original Image and and apply different PSF on every part named Figure 2(a)(b)(c)(d) A,B,C,D.

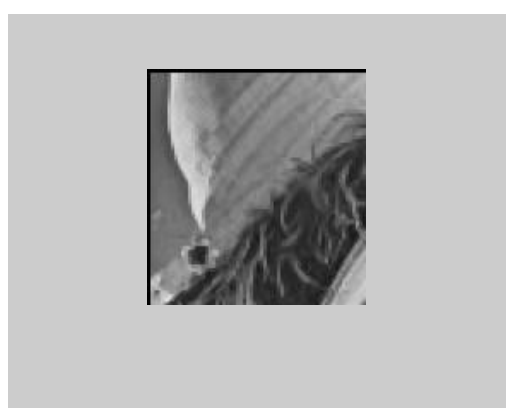

Figure 2(a) Original Image part A

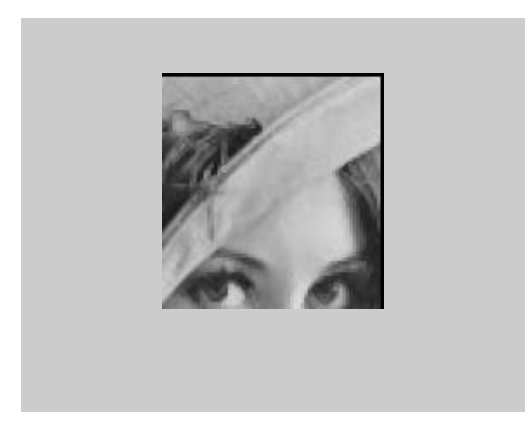

Figure 2(b) Original Image part B

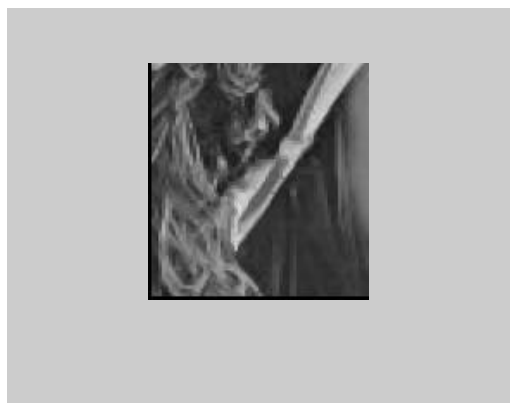

Figure 2(c) Original Image part C 


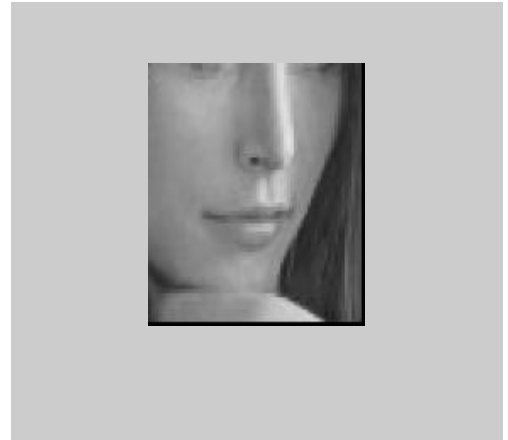

Figure 2(d) Original Image part $D$

Figure 3. (a)(b)(c)(d) Four divided original image. The following image is depicted as blurred images for all parts A,B,C,D.

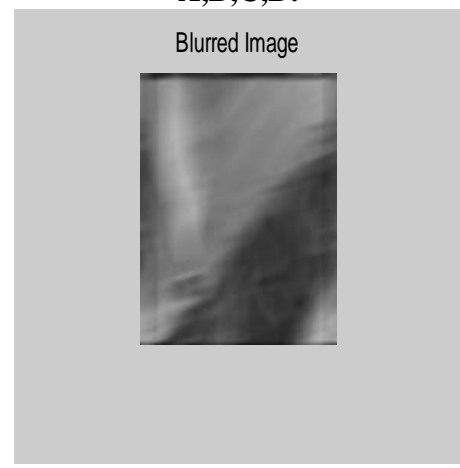

Figure 3(a) Blurred Image Part A

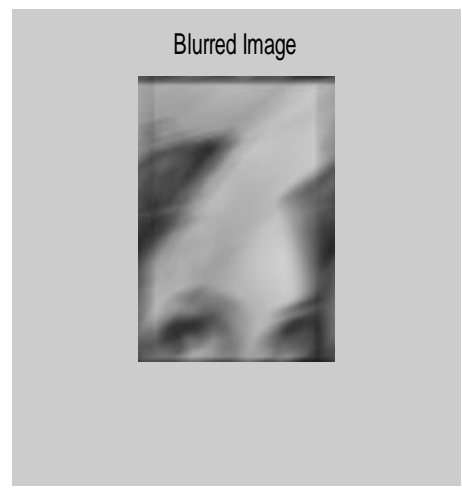

Figure 3(b) Blurred Image Part B

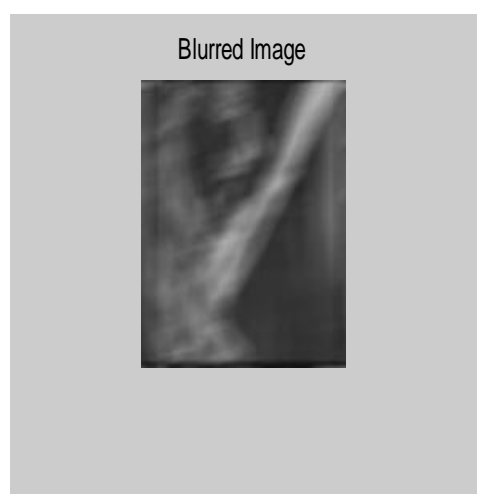

Figure 3(c) Blurred Image Part C

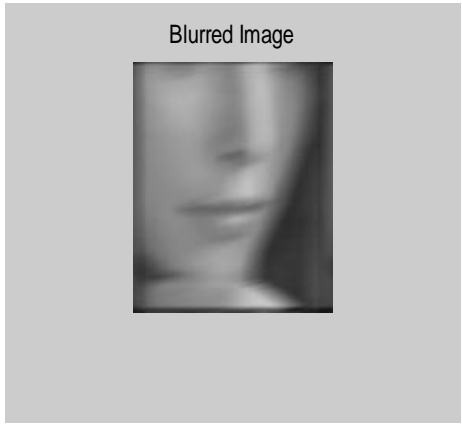

Figure 3(d) Blurred Image Part D

Figure 2(a)(b)(c)(d) Four divided blur image The sample image after applying the proposed algorithm will be as follows:

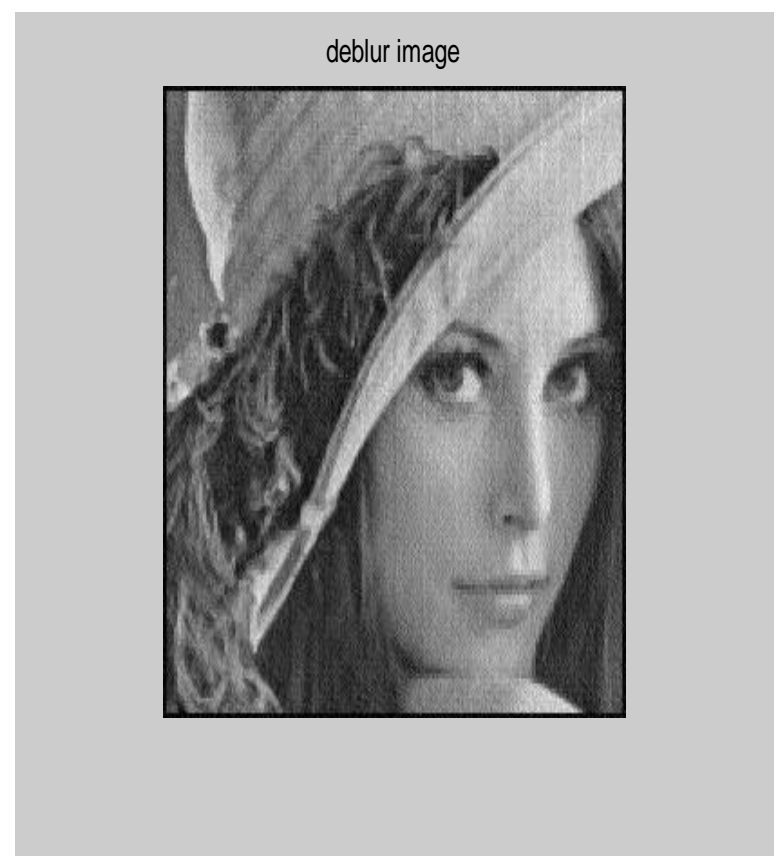

Figure 4 final image computed from our algorithm

The result of our algorithm is quite promising from parallel computation view. We can compute the each part independent from each other and final result will be combination of all divided images

\section{CONCLUSION}

This paper addresses the blind image restoration problem, Namely, given a number of different, blurred, and noisy PSF of a single ideal image, one wants to restore the original image. To solve this problem, a new parallel model is introduced. The restoration problem at hand in each of these approaches reduces to the problem of solving a multiple independent set of images on which algorithm is applied parallel. The algorithm is proposed that combines the benefits of the simple estimation and powerful parallel computation. An efficient iterative two-phase algorithm is presented for 
solving the defined problem, and convergence is assured to the optimal point.

The future work of this paper is to increase the speed of the deblurring process that is reducing thenumber of iteration using for deblurring the image for achieving better quality image.

\section{REFERENCES}

[1] Michael Elad and Arie Feuer, Senior Member, IEEE Restoration of a Single Superresolution Image from Several Blurred, Noisy, and Undersampled Measured Images

[2] WANG Shoujue, CAO Yu, HUANG Yi A Novel Image Restoration Approach Based on Point Location in Highdimensional Space Geometry

[3] Rajeev Srivastava*, Harish Parthasarthyt, JRP Guptat and D. Roy Choudharyl Image Restoration from Motion Blurred Image Using Pdes Formalism

[4] Dong-Dong Cao, Ping Guo Blind Image Restoration Based On Wavelet Analysis

[5] Gabriel Cristdbal And Rafael Navarro BLIND AND ADAPTIVE IMAGE RESTORATION IN THE FRAMEWORK OF A MULTISCALE GABOR REPRESENTATION
[6] R. L. Lagendijk and J. Biemond Iterative Identification and Restoration of Images. Boston, MA: Kluwer, 1991.

[7] D. C. Ghiglia, "Space-invariant deblurring given N independently blurred images of a common object," $J$. Opt. Soc. Amer., vol. 1, pp. 398-402, Apr. 1984.

[8] S. J. Ko and Y. H. Lee, "Nonlinear spatio-temporal noise suppression techniques with applications in image sequence processing," IEEE Int. Symp. CIS, 1991, vol. 5, pp. 662-665.

[9] Artemy Baxansky and Meir Tzur , Zoran Corporation, Haifa, Israel 2010 IEEE 26-th Convention of Electrical and Electronics Engineers in Israel

[10] Weisheng Dong, Lei Zhang, Member, IEEE, Guangming Shi, Senior Member, IEEE, and Xiaolin Wu, Fellow, IEEE. IEEE TRANSACTIONS ON IMAGE PROCESSING, VOL. 20, NO. 7, JULY 2011

[11] Ms.S.Ramya Kalasalingam University, Anand Nagar, Krishnankoil , PROCEEDINGS OF ICETECT 2011

[12] Ming Jiang, Ge Wang, Fellow, IEEE, Margaret W. Skinner, Jay T. Rubinstein, Member, IEEE, and Michael W. Vannier, Member, IEEE, IEEE TRANSACTIONS ON MEDICAL IMAGING, VOL. 22, NO. 7, JULY 2003

[13] Ta-Hsin Li, Member, IEEE, and Keh-Shin Lii, Member,IEEE 